\title{
Enhancing the Hot-Phonon Bottleneck Effect in a Metal Halide Perovskite by Terahertz Phonon Excitation
}

\author{
Fumiya Sekiguchi®, Hideki Hirori®, Go Yumoto®, Ai Shimazaki, Tomoya Nakamura๑, \\ Atsushi Wakamiya๑, and Yoshihiko Kanemitsu $\odot^{*}$ \\ Institute for Chemical Research, Kyoto University, Uji, Kyoto 611-0011, Japan
}

(Received 18 September 2020; accepted 13 January 2021; published 16 February 2021)

\begin{abstract}
We investigate the impact of phonon excitations on the photoexcited carrier dynamics in a lead-halide perovskite $\mathrm{CH}_{3} \mathrm{NH}_{3} \mathrm{PbI}_{3}$, which hosts unique low-energy phonons that can be directly excited by terahertz pulses. Our time-resolved photoluminescence measurements reveal that strong terahertz excitation prolongs the cooling time of hot carriers, providing direct evidence for the hot-phonon bottleneck effect. In contrast to the previous studies where phonons are treated as a passive heat bath, our results demonstrate that phonon excitation can significantly perturb the carrier relaxation dynamics in halide perovskites through the coupling between transverse- and longitudinal-optical phonons.
\end{abstract}

DOI: 10.1103/PhysRevLett.126.077401

Lead-halide perovskites are promising materials for future optoelectronic devices such as low-cost but highperformance solar cells and light-emitting diodes [1-6]. For such device development, fundamental knowledge of the dynamics of photoexcited carriers is indispensable [7,8]. Particularly, the dynamics of hot carriers, i.e., carriers with energies well above the band edge, is a crucial issue affecting device characteristics including photodetections $[9,10]$ and optical gains [11-13]. Most importantly, the concept of a hot-carrier solar cell has recently attracted extensive interest [14-16]; if the hot carriers can be utilized before they lose their excess energy via relaxation processes, it might lead to an efficiency beyond the ShockleyQueisser limit [17]. It has been reported that hot-carrier cooling in halide perovskites occurs on a slower timescale than in conventional inorganic semiconductors, and the origin of the long lifetime of hot carriers and methods to utilize them are under active discussion [18-21]. Because hot carriers lose their energy mainly via phonon emission $[7,8]$, the properties of phonons and electron-phonon interactions in halide perovskites should play a key role in the observed phenomena.

Halide perovskites possess unique phonon properties including strong anharmonicity [22-26] and low phonongroup velocity [27], which influence the characteristic aspects of these materials such as very low thermal conductivity $[25,28]$ and negative thermo-optic coefficients [29]. Furthermore, the optically active phonon modes, that

Published by the American Physical Society under the terms of the Creative Commons Attribution 4.0 International license. Further distribution of this work must maintain attribution to the author(s) and the published article's title, journal citation, and DOI. are related to the lead-halide cage, lie at low energies corresponding to terahertz (THz) frequencies [30-32]. In particular, $\mathrm{CH}_{3} \mathrm{NH}_{3} \mathrm{PbI}_{3}\left(\mathrm{MAPbI}_{3}\right)$, the most promising candidate for use in solar cells, has a strong phononabsorption resonance around $1 \mathrm{THz}$, which can be excited easily by a tabletop light source. This feature, together with an efficient electron-phonon coupling in halide perovskites [33], has stimulated investigations on the connection between phonon-mode excitation and optical properties, such as the optical band gap, in this material [34,35]. Concerning carrier relaxations, phonons have been dealt with as a passive heat bath so far [7,8,18,20,21]. Although studies of the impact of direct phonon excitation may provide new insights into the roles of carrier-phonon and phonon-phonon interactions in hot-carrier dynamics, to the best of our knowledge, no such studies have been performed until now.

In this study, we investigated the influence of a THzinduced phonon population on the hot-carrier relaxation dynamics in $\mathrm{MAPbI}_{3}$ by performing time-resolved photoluminescence (PL) measurements. Upon $\mathrm{THz}$ excitations, we observed an ultrafast quench of the total PL intensity and a simultaneous enhancement of the PL intensity on the high-energy side, revealing that long-lived hot carriers are generated by THz pulses. The cooling dynamics of the hot carriers depends on the THz-excitation intensity, with slower time constant at higher $\mathrm{THz}$ intensities under the same carrier density. This indicates that long-lived hot carriers appear as a result of a hot-phonon bottleneck (HPB) effect triggered by direct $\mathrm{THz}$ excitation of transverse-optical (TO) phonons.

Figure 1(a) shows a schematic picture of the PL measurement of a sample under excitation by an optical pulse and a THz pulse [36-39]. A 100-nm-thick $\mathrm{MAPbI}_{3}$ film sample was grown on a quartz substrate [40]. 


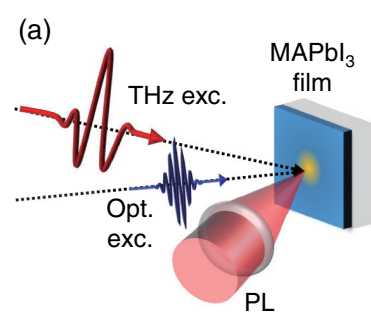

(c)

(b)

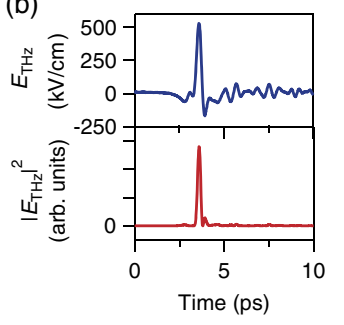

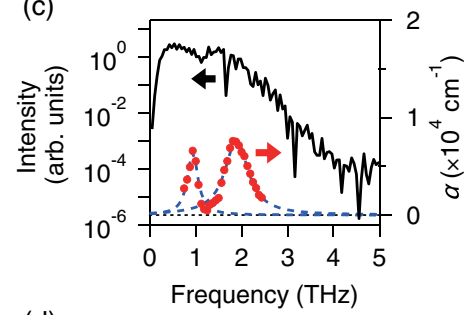

(d)

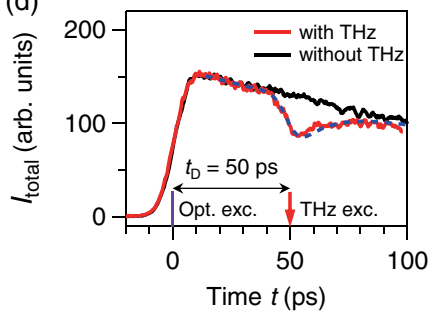

(a)

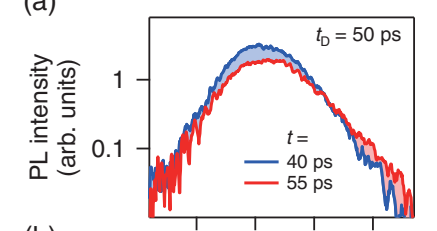

(b)

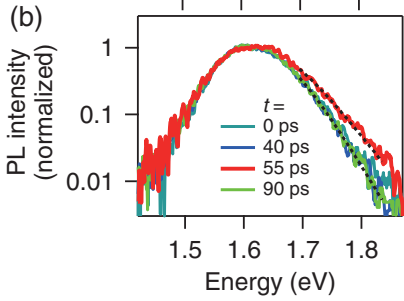

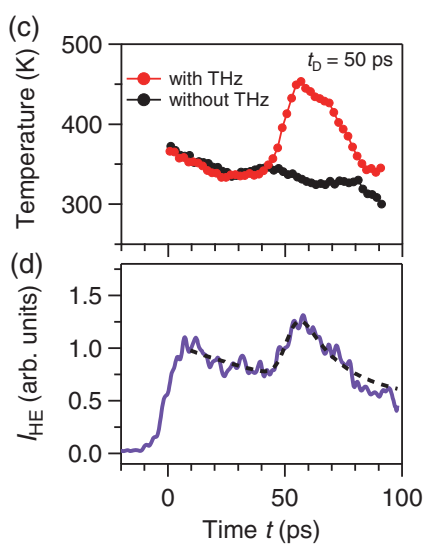

FIG. 2. Time evolutions of PL spectra and carrier temperature at the THz-excitation delay time of $t_{D}=50 \mathrm{ps}$. (a) PL spectra before (blue) and immediately after the $\mathrm{THz}$ excitation (red), i.e., at $t=40,55$ ps. For each spectrum, the PL counts of the streak data were integrated over a time period of $10 \mathrm{ps}$. (b) PL spectra at various times, i.e., at $t=0,40,55$, and $90 \mathrm{ps}$ normalized to their peak intensities. (c) Carrier temperatures as a function of time with and without $\mathrm{THz}$ excitation. (d) $I_{\mathrm{HE}}$ dynamics; PL intensity dynamics in the high $(1.8 \mathrm{eV})$-photon energy region. To obtain time traces with sufficient intensity, the PL counts were integrated over a spectral width of $0.1 \mathrm{eV}$.

$\sim 200 \mathrm{ps}$ through radiative and nonradiative carrier recombination processes. The $\mathrm{THz}$ electric field was set to the maximum amplitude $\left(E_{\mathrm{THz}}=530 \mathrm{kV} / \mathrm{cm}\right)$. The comparison of the two time traces clearly shows that the $I_{\text {total }}$ is quenched by the THz excitation within $\sim 10 \mathrm{ps,} \mathrm{which} \mathrm{is} \mathrm{on}$ the timescale of the instrument-response time. $I_{\text {total }}$ then recovers relatively slowly compared to the quench dynamics. Such an ultrafast quench and recovery of the PL intensity in picosecond timescale cannot be accounted for by a simple average heating of the entire sample, and requires a discussion on the dynamic interplay between the carrier and phonon subsystems.

To investigate the THz-induced change in the carrier distribution, we compare the PL spectra before and immediately after the $\mathrm{THz}$ excitation in Fig. 2(a). The two spectra were obtained by integrating the streak data over time windows of $40 \pm 5$ and $55 \pm 5$ ps, respectively. Figure 2(a) clearly shows that the THz-induced change in the PL spectrum is not uniform. To discuss the temporal evolution of the spectral shape, several normalized spectra obtained in the different time windows are shown in Fig. 2(b). It can be seen that the high-energy tail of the spectrum becomes less steep immediately after the $\mathrm{THz}$ excitation, indicating an increase in carrier temperature. The carrier temperature can be estimated by fitting this high-energy PL tail to a Boltzmann distribution function convoluted with the instrumental resolution function [40]. The obtained temporal evolution, as shown in Fig. 2(c), reveals that the carrier temperature increases by about $100 \mathrm{~K}$ upon $\mathrm{THz}$ excitation, and subsequently cools down 
to the same temperature as that without $\mathrm{THz}$ excitation. The temporal coincidence between the quench of $I_{\text {total }}$ [Fig. 1(d)] and the temperature rise [Fig. 2(c)] indicates that $I_{\text {total }}$ is suppressed for a short time due to carrier heating by the $\mathrm{THz}$ excitation. The $\mathrm{THz}$ excitation brings carriers to higher energy states with lower PL efficiencies, but subsequent carrier cooling by carrier-phonon interactions results in the recovery of $I_{\text {total }}$.

Furthermore, while $I_{\text {total }}$ is suppressed by the $\mathrm{THz}$ excitation, the PL intensity is enhanced in the high-energy region above $1.7 \mathrm{eV}$ as shown in Fig. 2(a). To investigate the high-energy PL intensity $\left(I_{\mathrm{HE}}\right)$ dynamics, we analyzed the transient of the PL integrated over a narrow $(0.1 \mathrm{eV})$ spectral region around $1.8 \mathrm{eV}$; Fig. 2(d) shows the enhancement in $I_{\mathrm{HE}}$ with a peak around $t=55 \mathrm{ps}$. Note that the intensity at $t=55 \mathrm{ps}$ is even larger than that at $t=0 \mathrm{ps}$. This feature demonstrates that the hot carriers induced by the $\mathrm{THz}$ excitation emit high-energy PL before they decay via nonradiative recombination or rapid cooling. As explained below, the observed $I_{\mathrm{HE}}$ dynamics is a manifestation of a slow carrier cooling induced by the $\mathrm{THz}$ excitation.

In $\mathrm{MAPbI}_{3}$, the carrier cooling time via electron-phonon coupling is normally shorter than 1 ps $[18,20,21,42]$. Although the initial state of the photoexcited carriers has a large excess energy of $1.48 \mathrm{eV}\left(=h \nu-E_{g}\right)$ in this experiment, rapid carrier cooling via electron-phonon coupling prevents the $I_{\mathrm{HE}}$ from having a significant amplitude. On the other hand, the $I_{\mathrm{HE}}$ is enhanced after the THz-induced carrier heating. This large $I_{\mathrm{HE}}$ amplitude in Fig. 2(d) does not drop as fast as the initial carrier cooling mentioned above. At this point, we should underline the fact that a relatively long $I_{\text {total }}$ recovery time was observed in Fig. 1(d), which indicates that a slow carrier cooling rate was induced by $\mathrm{THz}$ excitation. As shown by the dashed lines in Figs. 1(d) and 2(d), the $I_{\text {total }}$ recovery and $I_{\mathrm{HE}}$ decay dynamics can be reproduced by an exponential decay with a time constant of $\sim 15 \mathrm{ps}$ convoluted with the instrument-response time. This time constant is significantly longer than the reported value for the carrier cooling time of $\sim 0.2-0.6 \mathrm{ps}$ in the carrier density region similar to this experiment of $\sim 6 \times 10^{17} \mathrm{~cm}^{-3}$ $[18,20,21,42]$.

We investigated the origin of the slow carrier cooling by measuring the THz-excitation intensity dependence of the PL dynamics. Figure 3(a) shows the $I_{\text {total }}$ dynamics around $t=50 \mathrm{ps}$ that were obtained for different THz-excitation intensities. Obviously, the PL quench becomes less significant as $E_{\mathrm{THz}}$ decreases. Furthermore, the PL recovery time also depends on the THz-excitation intensity; $I_{\text {total }}$ recovers faster at lower $E_{\mathrm{THz}}$ values. Figure 3(b) plots the recovery time constant as a function of the THz-excitation intensity. Although the instrument-response time limits the resolution at the low intensity region, the PL recovery time clearly increases with $\mathrm{THz}$ intensity and exceeds $10 \mathrm{ps}$ at higher intensities above $\sim 1.5 \times 10^{-4} \mathrm{~J} / \mathrm{cm}^{2}$.
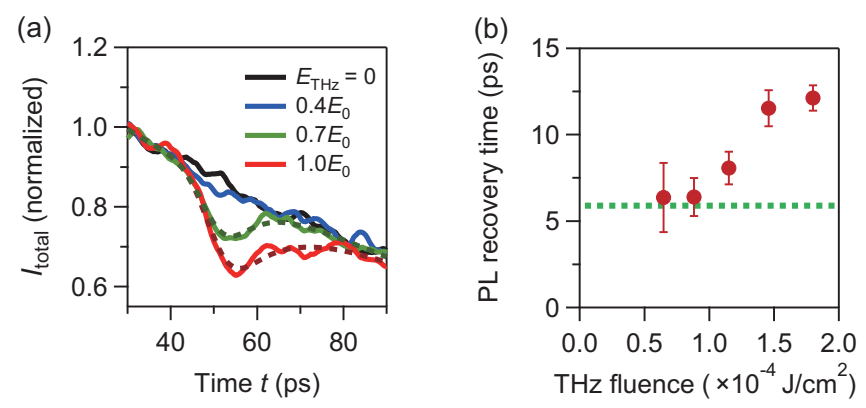

FIG. 3. PL recovery time prolonged by the $\mathrm{THz}$ excitation. THz-excitation intensity dependence of (a) $I_{\text {total }}$ dynamics for $t_{D}=50$ ps with $E_{0}=530 \mathrm{kV} / \mathrm{cm}$ and (b) the PL recovery time constant, where the dashed line around 6 ps indicates the time resolution of the streak camera.

Several mechanisms have been proposed to explain the slow cooling rates of hot carriers in halide perovskites, including polaron screening that protects carriers from phonon scattering [14,19] and Auger recombination inducing carrier heating [21]. Moreover, an effect attributed to the HPB has been reported in the high carrier density region $[18,20,43]$. The HPB effect denotes a phenomenon where highly populated phonon modes, mainly LO phonons interacting with the carriers, impede carrier cooling. This occurs because in addition to the phonon emission by the carriers, a large population of phonon modes increases the phonon-absorption rate which reheats the carriers. With respect to screening and Auger heating, their efficiencies should depend mainly on the carrier density. However, the carrier densities in Fig. 3(a) do not increase through application of the $\mathrm{THz}$ excitation. On the other hand, the THz-excitation spectrum is resonant with the lowfrequency $\mathrm{TO}$ phonon modes in $\mathrm{MAPbI}_{3}$ as shown in Fig. 1(c), and hence, a stronger THz excitation results in a higher phonon population. This is in line with the HPB mechanism; higher populations of phonon modes render the carrier cooling rate slower. From the linewidth of the phonon mode at $0.9 \mathrm{THz}$, the lifetime of the TO phonon population is estimated to be longer than $7 \mathrm{ps}$, which is consistent with the observed timescale of the THz-induced hot-carrier lifetime.

To confirm that direct excitation of TO phonons can induce slow cooling of hot carriers, we measured the PL dynamics under excitation conditions where the $\mathrm{THz}$ pulse irradiates the sample before the optical pulse, i.e., $t_{D}<0$. In contrast to the case of a positive delay time, this condition allows the excitation of only TO phonons and excludes the influences of carrier excitation. The red (blue) curve in Fig. 4(a) shows the dynamics of $I_{\text {total }}$ observed for $t_{D}=-3 \mathrm{ps}(-23 \mathrm{ps})$, while the black curve is data obtained without the THz excitation. To compare $I_{\text {total }}$ dynamics, each time trace is normalized to the value at $t=70 \mathrm{ps}$ where the influence of the THz excitation is negligible. In Fig. 4(a), the time trace for $t_{D}=-3$ ps shows that $I_{\text {total }}$ just after the 


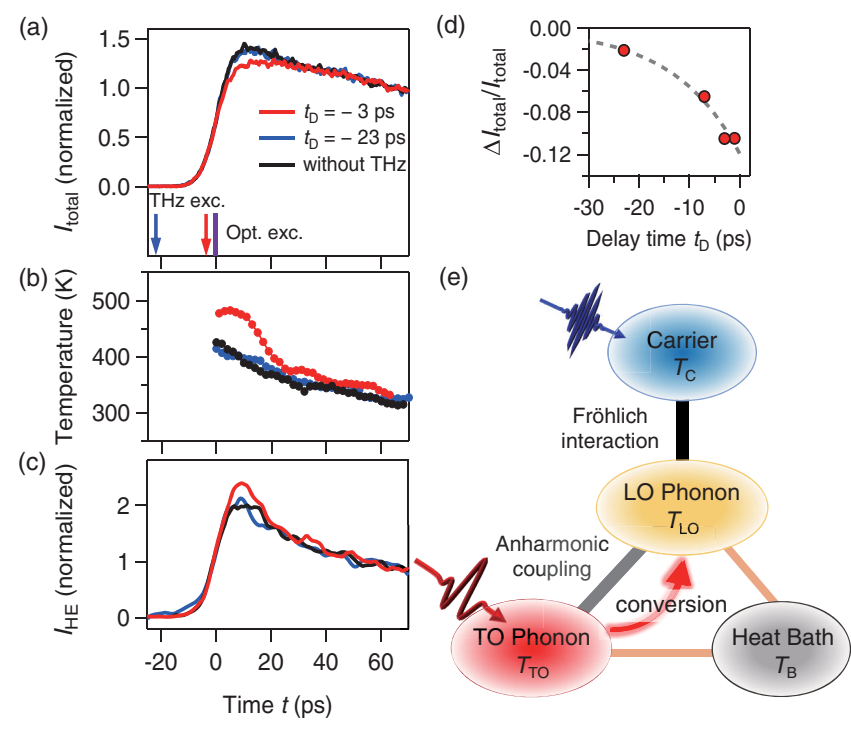

FIG. 4. PL modulation induced by THz excitations at negative delay times. (a) $I_{\text {total }}$ for negative delay times $\left(t_{D}=-3\right.$ and $-23 \mathrm{ps}$ ) and without the $\mathrm{THz}$ excitation. Each time trace is normalized to the value at $t=70 \mathrm{ps}$. The vertical purple solid line and red (blue) arrow indicate the time of the optical excitation and $\mathrm{THz}$ excitation at $t_{D}=-3 \mathrm{ps}(-23 \mathrm{ps})$, respectively. (b) The corresponding carrier-temperature dynamics. (c) $I_{\mathrm{HE}}$ dynamics; PL intensity dynamics in the high $(1.8 \mathrm{eV})$-photon energy region. (d) Delay time $t_{D}$ dependence of $I_{\text {total }}$ reduction at negative delay times. The values were obtained by integrating the data within the time range $t=0-20 \mathrm{ps}$. The reduction is measured relative to the data without $\mathrm{THz}$ excitation. (e) Phenomenological model of the thermal couplings between the subsystems: photoexcited carriers, LO phonons, TO phonons, and acoustic phonons.

photoexcitation (i.e., $t=0-20 \mathrm{ps}$ ) is reduced by the $\mathrm{THz}$ excitation. The corresponding carrier-temperature dynamics are shown in Fig. 4(b). The comparison of Figs. 4(a) and 4(b) clearly shows a correlation between the drop of $I_{\text {total }}$ and the carrier temperature; significantly higher temperatures are observed at times when $I_{\text {total }}$ of the red curve is below that of the black curve. The time evolution of the $I_{\mathrm{HE}}$ in Fig. 4(c) shows an enhancement in the high-energy PL intensity at early times before 20 ps. These observations reveal that direct excitation of TO phonons can alter the carrier distribution.

From the absorption spectrum shown in Fig. 1(c) and the incident THz-excitation fluence of $2.0 \times 10^{-4} \mathrm{~J} / \mathrm{cm}^{2}$, the energy injected into the sample is estimated to be $\sim 0.5 \mathrm{~J} / \mathrm{cm}^{3}$. If this energy is shared in the entire system, the average sample temperature estimated from the specific heat of $\mathrm{MAPbI}_{3}$ can increase only by $\sim 0.4 \mathrm{~K}$, which cannot explain a significant modulation of the carrier dynamics. Therefore, the enhanced hot carriers can appear only within the timescale before the population of the excited TO phonon-mode decays. This can be confirmed in Figs. 4(a)-4(c) showing that the $\mathrm{THz}$ excitation at $t_{D}=$ -23 ps hardly affects the PL dynamics. In Fig. 4(d), we plot the reduction of $I_{\text {total }}$ (relative to that without $\mathrm{THz}$ excitation) in the time range $t=0-20 \mathrm{ps}$ as a function of $t_{D}$. The figure shows that the $\mathrm{THz}$-induced hot-carrier effect decays with a time constant of 12 ps. This value is consistent with the carrier cooling time observed in the case of $t_{D}>0$, reflecting the decay time of the TO phonon population.

Figure 4(e) schematically shows how the THz excitation in this experiment can realize an artificial HPB condition. Usually, the dominant contribution to carrier cooling comes from the longitudinal-optical (LO) phonon emission due to the strong Fröhlich interaction between charged carriers and LO phonons $[7,8]$ [as indicated by the thick arrow in Fig. 4(e)], while the THz excitation excites optically active TO phonon modes [34,35]. Although a finite coupling between photoexcited carriers and TO phonon modes has been reported in halide perovskites [44-46], there is no evidence that negates the dominant role of LO phonons. On the other hand, large anharmonicity of phonon modes in halide perovskites, which stems from the soft $\mathrm{Pb}$-I cage that is hybridized with the MA cation motions, introduces considerable couplings among different phonon modes $[22,25,43]$. Through such an anharmonic coupling, it is possible that $\mathrm{THz}$ excitation induces a direct phonon population transfer [47] from excited TO phonons to LO phonons. Another possible pathway is an indirect TO-toLO phonon conversion mediated by the acoustic phonons; the TO phonon population excited by $\mathrm{THz}$ pulses decays into acoustic phonons and then up-conversion of acoustic phonons occurs generating LO phonon population as proposed in Ref. [43]. These pathways coexist, and an effective coupling between TO and LO phonon modes can be considered. In this way, the THz-pulse-induced TO phonon population is transferred to LO phonons [Fig. 4(e)], leading to the longer LO phonon lifetime and a slow cooling of hot carriers. Indeed, with using a phenomenological temperature model of thermally coupled subsystems, i.e., carriers, LO and TO phonons, we can qualitatively reproduce the HPB effect through the increase of the TO phonon temperature upon the THz excitation, as described in the Supplemental Material [40]. In the previous studies, the onset of the HPB was observed by increasing the density or energy of the photoexcited carriers $[18,20,42,43,48,49]$. In contrast, here we realized a slow cooling of hot carriers through intentional excitation of phonons at the fixed carrier density. Though sophisticated theoretical works are needed to provide more quantitative discussions about the efficiency of phonon-conversion pathways, these observations provide direct evidence for the working principle of HPB.

To summarize, in this study we have shown that hot carriers are generated in a $\mathrm{MAPbI}_{3}$ thin film by $\mathrm{THz}$ excitation, evidenced by the enhanced high-energy PL intensity. The cooling rate of the hot carriers depends on the THz-excitation intensity, and a slow time constant of more than 10 ps can be achieved under strong $\mathrm{THz}$ 
excitation above $1.5 \times 10^{-4} \mathrm{~J} / \mathrm{cm}^{2}$. Taking into account the fact that a slow carrier cooling has also been observed when the THz pulse arrives before the optical excitation, where $\mathrm{THz}$ pulses excite only TO phonon modes, a hot-phonon bottleneck effect has been shown to occur under the direct excitation of the TO phonon modes via TO-LO phonon coupling. From a broader perspective, this study has demonstrated that the excitation of a phonon system, which is normally treated as a passive heat bath, can be used as a new degree of freedom to modulate the carrier dynamics. Combined with phonon-mode engineering technologies enabled by elemental substitution or nanostructuring, intentional excitation of phonon modes will be a new scheme to control carrier dynamics and utilize carrier energies through controlled perturbation of phonon modes.

We thank T. Handa, T. Yamada, and H. Tahara for fruitful discussions. Part of this study was supported by JSPS KAKENHI Grant No. JP19H05465.

*Corresponding author. kanemitu@scl.kyoto-u.ac.jp

[1] A. Kojima, K. Teshima, Y. Shirai, and T. Miyasaka, J. Am. Chem. Soc. 131, 6050 (2009).

[2] S. D. Stranks and H. J. Snaith, Nat. Nanotechnol. 10, 391 (2015).

[3] B. R. Sutherland and E. H. Sargent, Nat. Photonics 10, 295 (2016).

[4] Y. Kanemitsu, J. Mater. Chem. C 5, 3427 (2017).

[5] M. M. Lee, J. Teuscher, T. Miyasaka, T. N. Murakami, and H. J. Snaith, Science 338, 643 (2012).

[6] Z.-K. Tan, R. S. Moghaddam, M. L. Lai, P. Docampo, R. Higler, F. Deschler, M. Price, A. Sadhanala, L. M. Pazos, D. Credgington, F. Hanusch, T. Bein, H. J. Snaith, and R. H. Friend, Nat. Nanotechnol. 9, 687 (2014).

[7] P. Y. Yu and M. Cardona, Fundamentals of Semiconductors (Springer, Berlin, 1996).

[8] J. Shah, Solid State Electron. 21, 43 (1978).

[9] M. Freitag, T. Low, F. Xia, and P. Avouris, Nat. Photonics 7, 53 (2013).

[10] Y.-F. Lao, A. G. U. Perera, L. H. Li, S. P. Khanna, E. H. Linfield, and H. C. Liu, Nat. Photonics 8, 412 (2014).

[11] B. N. Gomatam and A.P. DeFonzo, IEEE J. Quantum Electron. 26, 1689 (1990).

[12] G. Yumoto, H. Tahara, T. Kawawaki, M. Saruyama, R. Sato, T. Teranishi, and Y. Kanemitsu, J. Phys. Chem. Lett. 9, 2222 (2018).

[13] E. Kobiyama, H. Tahara, R. Sato, M. Saruyama, T. Teranishi, and Y. Kanemitsu, Nano Lett. 20, 3905 (2020).

[14] P. P. Joshi, S. F. Maehrlein, and X. Zhu, Adv. Mater. 31, 1803054 (2019).

[15] S. Kahmann and M. A. Loi, J. Mater. Chem. C 7, 2471 (2019).

[16] M. Li, J. Fu, Q. Xu, and T. C. Sum, Adv. Mater. 31, 1802486 (2019).

[17] R. T. Ross and A. J. Nozik, J. Appl. Phys. 53, 3813 (1982).
[18] Y. Yang, D. P. Ostrowski, R. M. France, K. Zhu, J. van de Lagemaat, J. M. Luther, and M. C. Beard, Nat. Photonics 10, 53 (2016).

[19] H. Zhu, K. Miyata, Y. Fu, J. Wang, P. P. Joshi, D. Niesner, K. W. Williams, S. Jin, and X.-Y. Zhu, Science 353, 1409 (2016).

[20] M. B. Price, J. Butkus, T. C. Jellicoe, A. Sadhanala, A. Briane, J. E. Halpert, K. Broch, J. M. Hodgkiss, R. H. Friend, and F. Deschler, Nat. Commun. 6, 8420 (2015).

[21] J. Fu, Q. Xu, G. Han, B. Wu, C. H. A. Huan, M. L. Leek, and T. C. Sum, Nat. Commun. 8, 1300 (2017).

[22] C. Katan, A. D. Mohite, and J. Even, Nat. Mater. 17, 377 (2018).

[23] A. N. Beecher, O. E. Semonin, J. M. Skelton, J. M. Frost, M. W. Terban, H. Zhai, A. Alatas, J. S. Owen, A. Walsh, and S. J. L. Billinge, ACS Energy Lett. 1, 880 (2016).

[24] F. Brivio, J. M. Frost, J. M. Skelton, A. J. Jackson, O. J. Weber, M. T. Weller, A. R. Goñi, A. M. A. Leguy, P. R. F. Barnes, and A. Walsh, Phys. Rev. B 92, 144308 (2015).

[25] A. Gold-Parker, P. M. Gehring, J. M. Skelton, I. C. Smith, D. Parshall, J. M. Frost, H. I. Karunadasa, A. Walsh, and M. F. Toney, Proc. Natl. Acad. Sci. U.S.A. 115, 11905 (2018).

[26] C. Gehrmann and D. A. Egger, Nat. Commun. 10, 3141 (2019).

[27] A. C. Ferreira, A. Létoublon, S. Paofai, S. Raymond, C. Ecolivet, B. Rufflé, S. Cordier, C. Katan, M. I. Saidaminov, A. A. Zhumekenov, O. M. Bakr, J. Even, and P. Bourges, Phys. Rev. Lett. 121, 085502 (2018).

[28] A. Pisoni, J. Jaćimović, O. S. Barišić, M. Spina, R. Gaál, L. Forró, and E. Horváth, J. Phys. Chem. Lett. 5, 2488 (2014).

[29] T. Handa, H. Tahara, T. Aharen, and Y. Kanemitsu, Sci. Adv. 5, eaax0786 (2019).

[30] C. La-o-vorakiat, H. Xia, J. Kadro, T. Salim, D. Zhao, T. Ahmed, Y. M. Lam, J.-X. Zhu, R. A. Marcus, M.-E. Michel-Beyerle, and E. E. M. Chia, J. Phys. Chem. Lett. 7, 1 (2016).

[31] M. Nagai, T. Tomioka, M. Ashida, M. Hoyano, R. Akashi, Y. Yamada, T. Aharen, and Y. Kanemitsu, Phys. Rev. Lett. 121, 145506 (2018).

[32] R. Sharma, M. Menahem, Z. Dai, L. Gao, T. M. Brenner, L. Yadgarov, J. Zhang, Y. Rakita, R. Korobko, I. Pinkas, A. M. Rappe, and O. Yaffe, Phys. Rev. Mater. 4, 051601 (R) (2020).

[33] M. Sendner, P. K. Nayak, D. A. Egger, S. Beck, C. Müller, B. Epding, W. Kowalsky, L. Kronik, H. J. Snaith, A. Pucci, and R. Lovrinčić, Mater. Horizons 3, 613 (2016).

[34] H. Kim, J. Hunger, E. Cánovas, M. Karakus, Z. Mics, M. Grechko, D. Turchinovich, S. H. Parekh, and M. Bonn, Nat. Commun. 8, 687 (2017).

[35] Z. Liu, C. Vaswani, L. Luo, D. Cheng, X. Yang, X. Zhao, Y. Yao, Z. Song, R. Brenes, R. J. H. Kim, J. Jean, V. Bulović, Y. Yan, K.-M. Ho, and J. Wang, Phys. Rev. B 101, 115125 (2020).

[36] K.-L. Yeh, M. C. Hoffmann, J. Hebling, and K. A. Nelson, Appl. Phys. Lett. 90, 171121 (2007).

[37] H. Hirori, A. Doi, F. Blanchard, and K. Tanaka, Appl. Phys. Lett. 98, 091106 (2011). 
[38] H. Hirori, K. Shinokita, M. Shirai, S. Tani, Y. Kadoya, and K. Tanaka, Nat. Commun. 2, 594 (2011).

[39] A. T. Tarekegne, K. Iwaszczuk, M. Zalkovskij, A. C. Strikwerda, and P. U. Jepsen, New J. Phys. 17, 043002 (2015).

[40] See Supplemental Material at http://link.aps.org/ supplemental/10.1103/PhysRevLett.126.077401 for the details of sample preparation, estimation of the carrier temperature, and simulation results based on the three temperature model, which includes Ref. [7].

[41] Y. Yamada, T. Nakamura, M. Endo, A. Wakamiya, and Y. Kanemitsu, Appl. Phys. Express 7, 032302 (2014).

[42] T. R. Hopper, A. Gorodetsky, J. M. Frost, C. Müller, R. Lovrincic, and A. A. Bakulin, ACS Energy Lett. 3, 2199 (2018).

[43] J. Yang, X. Wen, H. Xia, R. Sheng, Q. Ma, J. Kim, P. Tapping, T. Harada, T. W. Kee, F. Huang, Y.-B. Cheng, M. Green, A. Ho-Baillie, S. Huang, S. Shrestha, R. Patterson, and G. Conibeer, Nat. Commun. 8, 14120 (2017).
[44] C. Wehrenfennig, M. Liu, H. J. Snaith, M. B. Johnston, and L. M. Herz, Energy Environ. Sci. 7, 2269 (2014).

[45] D. Zhao, H. Hu, R. Haselsberger, R. A. Marcus, M.-E. Michel-Beyerle, Y. M. Lam, J.-X. Zhu, C. La-o-vorakiat, M. C. Beard, and E. E. M. Chia, ACS Nano 13, 8826 (2019).

[46] L. Luo, L. Men, Z. Liu, Y. Mudryk, X. Zhao, Y. Yao, J. M. Park, R. Shinar, J. Shinar, K.-M. Ho, I. E. Perakis, J. Vela, and J. Wang, Nat. Commun. 8, 15565 (2017).

[47] M. Kozina, M. Fechner, P. Marsik, T. van Driel, J. M. Glownia, C. Bernhard, M. Radovic, D. Zhu, S. Bonetti, U. Staub, and M. C. Hoffmann, Nat. Phys. 15, 387 (2019).

[48] Y. Rosenwaks, M. C. Hanna, D. H. Levi, D. M. Szmyd, R. K. Ahrenkiel, and A. J. Nozik, Phys. Rev. B 48, 14675 (1993).

[49] W. S. Pelouch, R. J. Ellingson, P. E. Powers, C. L. Tang, D. M. Szmyd, and A. J. Nozik, Phys. Rev. B 45, 1450 (1992). 